\title{
A manifold of pure Gibbs states of the Ising model on a Cayley tree
}

\author{
Daniel Gandolfo, Jean Ruiz \\ Centre de Physique Théorique, Aix-Marseille Univ, \\ CNRS UMR 7332, Univ Sud Toulon Var, \\ 13288 Marseille cedex 9, France; \\ Senya Shlosman \\ Centre de Physique Théorique, Aix-Marseille Univ, \\ CNRS UMR 7332, Univ Sud Toulon Var, \\ 13288 Marseille cedex 9, France; \\ Inst. of the Information Transmission Problems, \\ RAS, Moscow, Russia
}

July 5, 2012

\begin{abstract}
We study the Ising model on a Cayley tree. A wide class of new Gibbs states is exhibited.
\end{abstract}

\section{Introduction}

In this paper we consider the well-studied n.n. Ising model on the Bethe lattice $\mathcal{T}^{k}$ (or Cayley tree). The natural title of the present paper would be "New Gibbs states for the Ising model on the Bethe lattice". However the subject of "New States on the Bethe lattice" is not new for already a long time, and was discussed by many authors. So a better name might be "All pure low-temperature states" (especially if it describes correctly what follows). 
In spite of its simplicity, some questions in the area of statistical mechanics on trees are still open, and new phenomena are encountered.

The $(+)$ and $(-)$ phases of the Ising model on the Bethe lattice - the translation invariant states $\mu^{+}$and $\mu^{-}-$are known to be extremal below the critical temperature $T_{c}$. An uncountable number of non-translation invariant pure states $\mu^{ \pm}$was constructed by Blekher and Ganikhodzhaev in [BG]. These states describe the coexistence of the $\mu^{+}$and $\mu^{-}$states along different 'rigid interfaces' $S$, analogous to the Dobrushin states in the 3D Ising model. One should think about $S$ as a path, connecting two points 'at infinity' of $\mathcal{T}^{k}$. In what follows we will call these states as BG-states and denote them also by $\mu_{S}^{ \pm}$.

The "free state" $\mu^{0}$, i.e. the one corresponding to 'zero' or 'empty' boundary condition is extremal in the interval below the critical temperature $T_{c}$ and above the critical temperature of the corresponding spin-glass model (see [BRZ]). In particular, it is not extremal at low temperatures. One does not know the decomposition of $\mu^{0}$ into extremal states (a question of A. van Enter). We will formulate below our conjecture about this decomposition.

Later Rakhmatullaev and Rozikov [RR introduced some new states, which they call weakly periodic states. Their construction is somewhat indirect and uses various subgroups of the free group.

In what follows we present an alternative construction of weakly periodic states, as well as the construction of many more pure states. This construction is a result of our attempt to understand the claim of [RR].

\section{New ground state configurations}

Let $\mathcal{T}^{k}=(V, E)$ be the uniform Cayley tree, where each vertex has $k+1$ neighbors. Let $0 \in V$ be its root. We consider the n.n. ferromagnetic Ising model on $\mathcal{T}^{k}$ with interaction $J>0$.

The following simple remark, valid for the Ising model on any rooted tree, will be very helpful.

Let $D \subset E$ be an arbitrary collection (finite or infinite) of edges of $\mathcal{T}^{k}$. Define the configurations $\sigma^{D+}, \sigma^{D-}$ on $\mathcal{T}^{k}$, which satisfy the property: for every bond $b \in E, b=\left(b_{1}, b_{2}\right) \subset V$

$$
\sigma_{b_{1}}^{D+} \sigma_{b_{2}}^{D+}=\sigma_{b_{1}}^{D-} \sigma_{b_{2}}^{D-}= \begin{cases}-1 & \text { for } b \in D, \\ +1 & \text { for } b \notin D,\end{cases}
$$




$$
\sigma_{0}^{D+}=+1, \sigma_{0}^{D-}=-1 .
$$

The existence and uniqueness of the configurations $\sigma^{D+}$ and $\sigma^{D-}$ is immediate. Note that the map $D \rightsquigarrow \sigma^{D+}$ is a one-to-one map between the subsets $D \subseteq E$ and the spin configurations on $\mathcal{T}^{k}$ which have the value +1 at the root $0 \in V$.

Suppose a set $D \subseteq E$ is given. For every vertex $v \in V$ define $d_{D}(v)$ to be the number of bonds in $D$, which are incident to $v$. Define the number

$$
d_{D}=\max _{v} d_{D}(v)
$$

Suppose now that the set $D$ is such that

$$
d_{D}<(k-1) / 2 \text {. }
$$

(For example, if all bonds in $D$ are disjoint, then $d_{D}=1$, in which case $k$ has to be at least 4). Then the following theorem holds.

Theorem 1 The configurations $\sigma^{D+}$ and $\sigma^{D-}$ are ground state configurations.

Let $\sigma$ be a ground state configuration, and $C \subset V$ be a connected finite set. The configuration $\sigma_{C}$, obtained from $\sigma$ by flipping all the spins of $\sigma$ in $C$, will be called a connected excitation of $\sigma$. We will call the ground state configuration $\sigma$ stable (see [DS]), if for any $v \in V$, any excess energy level $E>0$, the number of connected excitations $\sigma_{C}$ of $\sigma$, satisfying

$$
H\left(\sigma_{C}\right)-H(\sigma)<E, \quad v \in C
$$

is bounded uniformly in $v$.

Theorem 2 Under the condition (1) the ground state configurations $\sigma^{D+}$ and $\sigma^{D-}$ are stable. 


\section{New low-T Gibbs states}

Denote by $\mu^{D_{+}}$and $\mu^{D_{-}}$the Gibbs states corresponding to the boundary conditions $\sigma^{D+}$ and $\sigma^{D-}$. The quantification of the last statement allows us to show the following

Theorem 3 There exists a value $T_{k}$ of the temperature, such that for all temperatures $T<T_{k}$ and all collections $D$ satisfying (1) the states $\mu^{D_{+}}$and $\mu^{D_{-}}$are extremal Gibbs states. (Here the temperature $T_{k}$ does not depend on $D)$.

These low-temperature Gibbs states are small perturbations of the ground states $\sigma^{D+}$ and $\sigma^{D-}$. It is interesting to observe that the free energy of the states $\mu^{D+}$ and $\mu^{D-}$ is higher than that of the states $\mu^{+}, \mu^{-}$and $\mu^{ \pm}$.

\section{Examples and comments}

1. $D=\varnothing$. In that case the states $\mu^{\varnothing+}$ and $\mu^{\varnothing-}$ are just the $(+)$ and $(-)$ states.

2. In the case when $D$ consists from a single bond $b$, the states $\mu^{b+}$ and $\mu^{b-}$ are among the non-translation invariant states $\mu_{S}^{ \pm}$constructed by Blekher and Ganikhodzhaev in [BG]. The interface curve $S$ in that case is just the curve intersecting $\mathcal{T}^{k}$ along the single bond $b$. The same is true for every finite collection $D$. Other states constructed in [BG] correspond to some countable families $D$.

3. Consider the case when $D=\bar{D}$ - a dimer covering of $\mathcal{T}^{k}$. That means that for every vertex $v \in V$ there exists exactly one bond $d(v) \in \bar{D}$, such that $v \in d(v)$. The existence of dimer coverings is straightforward. 


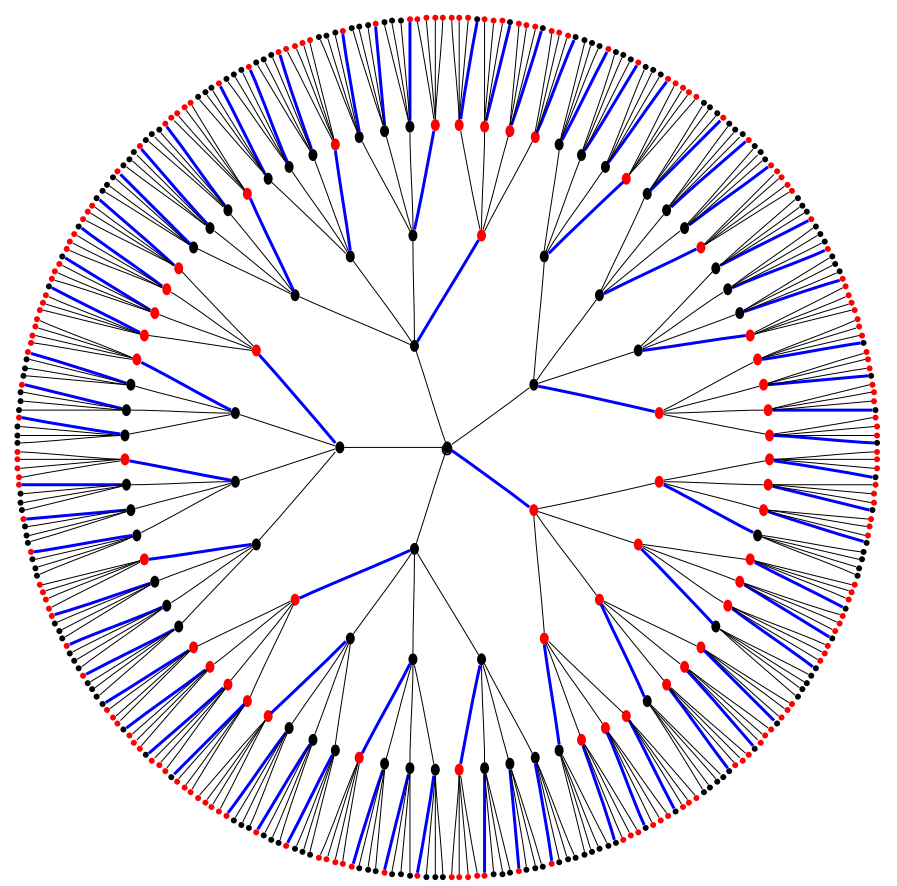

Fig. 1. The configuration $\sigma^{\bar{D}+}$ on the Cayley tree $\mathcal{T}^{4}$ (for four generations). Black and red dots correspond to $(-)$ and $(+)$ spins.

Here $\bar{D}$ is a dimer covering of $\mathcal{T}^{4}$ (blue bonds).

In the terminology of $\left[\mathrm{RR}\right.$ the ground state configurations $\sigma^{\bar{D}+}$ and $\sigma^{\bar{D}-}$ are called weakly periodic. According to our theorems they generate the corresponding low-temperature extremal states $\mu^{\bar{D}+}$ and $\mu^{\bar{D}-}$. The qualitative difference between the states $\mu^{\bar{D}} \pm$ and the BG states $\mu_{S}^{ \pm}$is the following. The states $\mu_{S}^{ \pm}$describe the coexistence of the $(+)$ and $(-)$ states separated by an interface $S$; that means for example that for every $R>0$ one can find (many) points $x \in V$, such that the 
phase in the ball $U_{R}(x)$ looks as a small perturbation of the $(+)$ or $(-)$ state. Namely, any point $x \in V$ with dist $(x, S)>(1+\varepsilon) R$ would go. On the other hand, the typical configuration of the state $\mu^{\bar{D}+}$ or $\mu^{\bar{D}-}$ around any point $x \in V$ has certain fractions of $(+)$ and $(-)$ spins.

4. Let $f_{D}(\beta)$ be the free energy of the Gibbs state of our Ising model at inverse temperature $\beta$, corresponding to the boundary conditions $\sigma^{D \pm}$. Unlike the classical case of the $\mathbb{Z}^{\nu}$ lattices, the free energy of the Ising model on $\mathcal{T}^{k}$ might depend on the boundary conditions. Clearly, for every $\beta$

$$
f_{\bar{D}}(\beta) \geq f_{D}(\beta) \geq f_{\varnothing}(\beta),
$$

moreover,

$$
f_{\bar{D}}(\beta)>f_{\varnothing}(\beta)
$$

for $\beta$ large enough. We conjecture that the states $\mu^{\bar{D}+}$ and $\mu^{\bar{D}-}$ have the highest possible free energy among all the Gibbs states at a given temperature on the tree $\mathcal{T}^{k}$ with $k=4$.

5. A more elaborate example is the 'secondary dimer covering', corresponding to a dimer covering $\bar{D}$. By this we mean any collection of bonds $\tilde{D}$, such that

- every bond $b \in \tilde{D}$ is incident to exactly two bonds $d^{\prime}, d^{\prime \prime}$ from $\bar{D}$;

- every bond $d \in \bar{D}$ is incident to exactly one bond $b$ from $\tilde{D}$. 


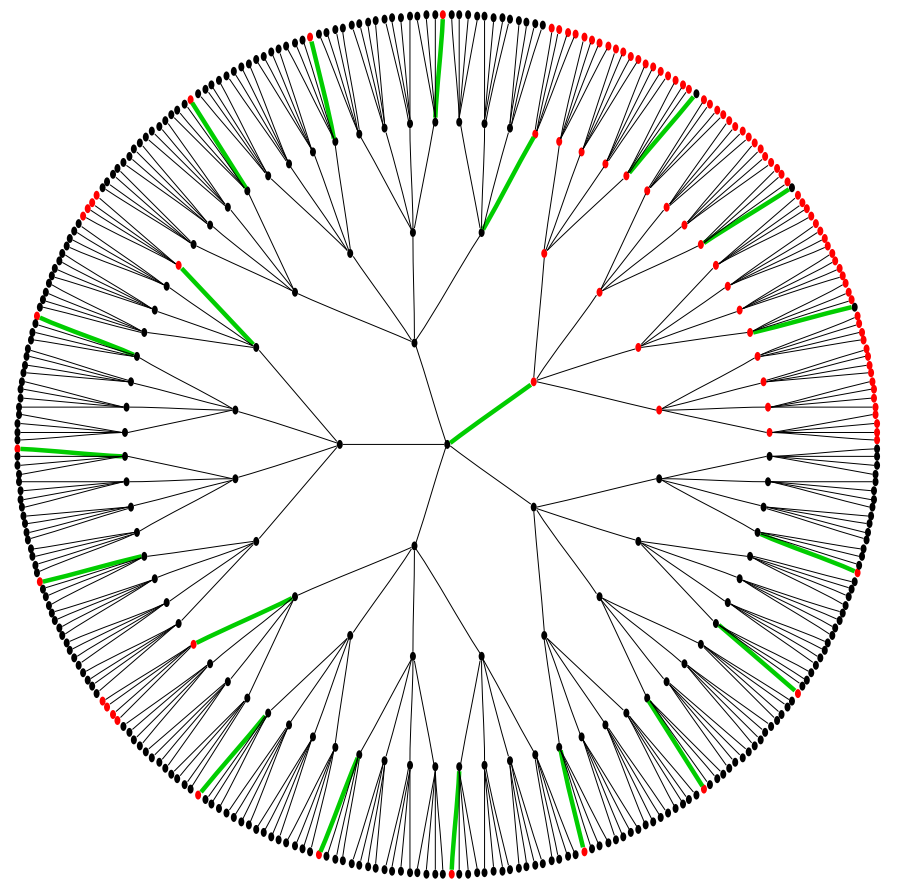

Fig. 2. Secondary dimer covering $\tilde{D}$ (green bonds) on the Cayley tree $\mathcal{T}^{4}$ (for four generations) corresponding to the primary dimer covering $\bar{D}$ (blue bonds)

of Figure 1.

Again, for low temperatures we have

$$
f_{\bar{D}}(\beta)>f_{\tilde{D}}(\beta)>f_{\varnothing}(\beta) .
$$

6. Yet another example can be obtained by considering the family $D^{*}$, corresponding to the following monomer-dimer covering. By this we mean (see Fig. 3) the collection of bonds $b \in E$, such that

- for every $b^{\prime} \neq b^{\prime \prime} \in D^{*}$ the distance $\operatorname{dist}\left(b^{\prime}, b^{\prime \prime}\right) \geq 2$, 
- for every $v \in V$ the distance $\operatorname{dist}\left(v, D^{*}\right) \leq 1$.

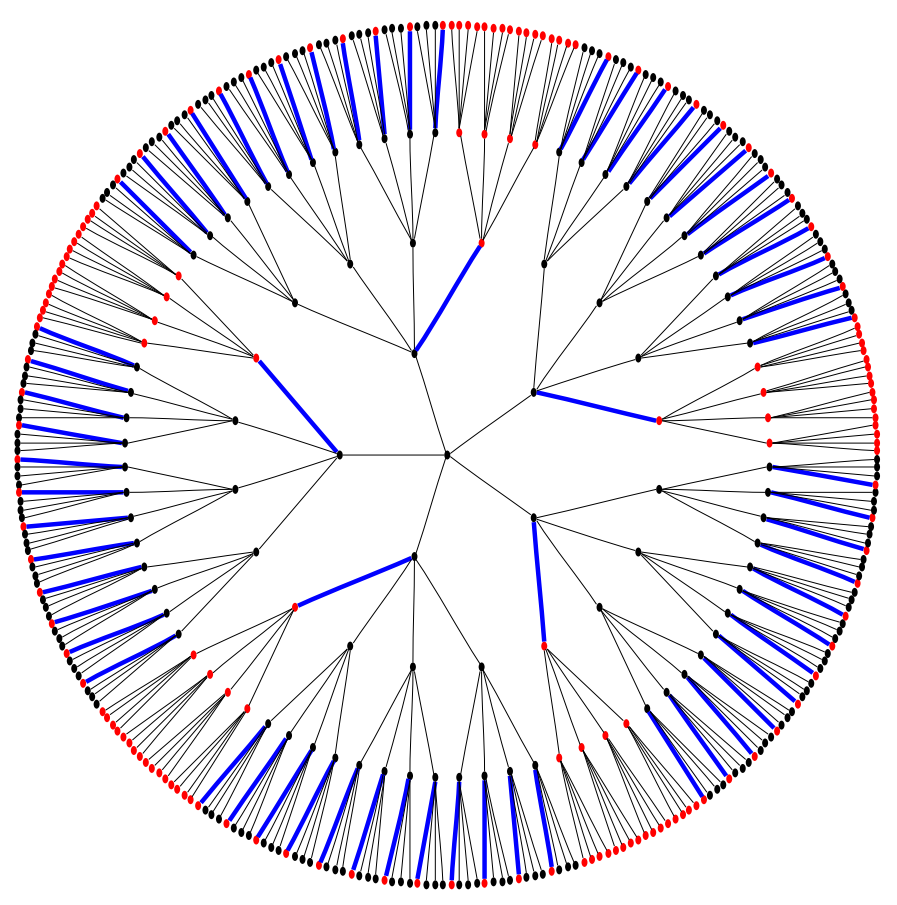

Fig. 3. Monomer-dimer covering.

7. A final example is the family $\hat{D}$, corresponding to the 'path covering' of the tree $\mathcal{T}^{k}$. Namely, the family $\hat{D}$ is defined by the property that every $v \in V$ belongs to precisely two bonds from $\hat{D}$. Thus the bonds from $\hat{D}$ split into double infinite non-intersecting paths, passing through each vertex of $\mathcal{T}^{k}$. To apply our results we need in this case $k \geq 6$. 


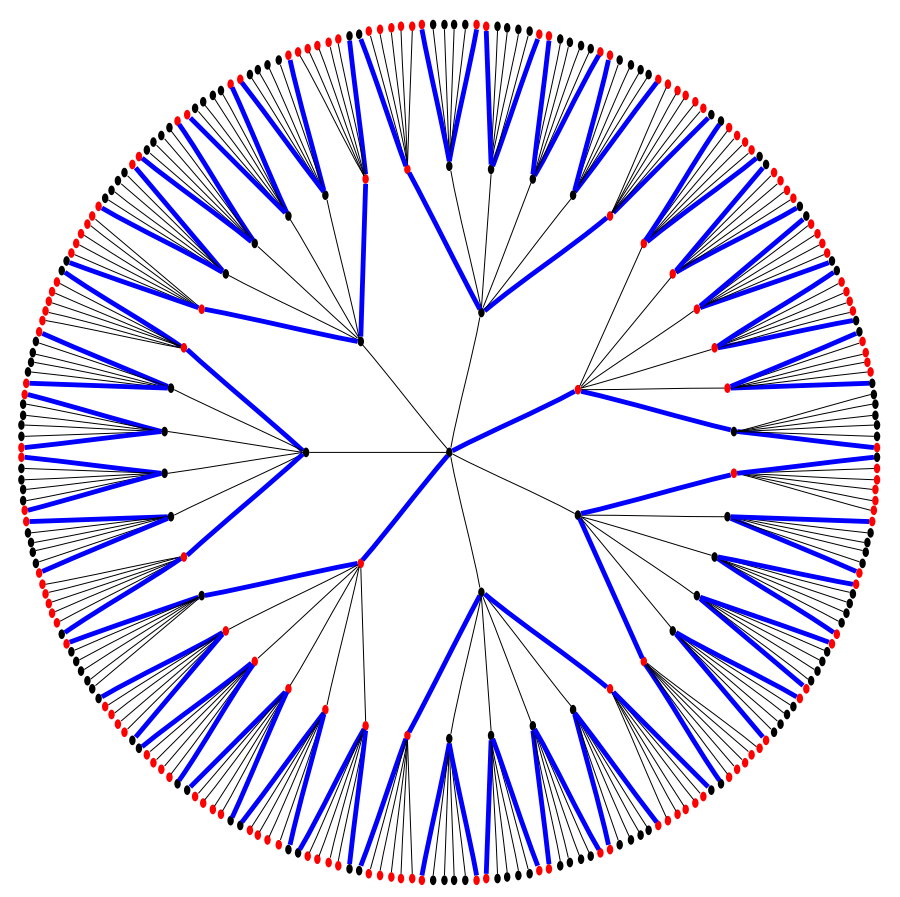

Fig. 4. Path covering of the tree $\mathcal{T}^{6}$.

As the reader can easily see, many more regular, as well as infinitely more irregular examples can be constructed in the manner above.

8. Next, we formulate our conjecture about the decomposition of the low temperature free state $\mu^{0}$ into extremal states. We think that this decomposition should be into our measures $\mu^{D_{+}}$and $\mu^{D_{-}}$with various sets $D$ which are sparse enough (depending on the temperature). 


\section{The proofs}

The proof of Theorem 3 is based on contour representation of the model. The remaining theorems follow from it.

Consider a configuration $\sigma$ that coincides with a ground state configuration $\sigma^{D}$ outside finite box $V$. A site $x \in V$ is called incorrect, iff $\sigma_{x} \neq \sigma_{x}^{D}$. The set $J$ of incorrect sites then decomposes into maximal connected components, $J=I_{1} \cup I_{2} \cup \cdots \cup I_{n}$. (Here a set of sites is called connected if for any two sites $x, y$ there exist a sequence $x=x_{1}, x_{2}, \ldots, x_{j}, \ldots x_{m}=y$ for which $x_{j}$ and $x_{j+1}$ are n.n. for all $i=1, \ldots, m-1$.)

Let us also introduce the external boundary $\partial_{E} I$ of $I$ to be the set of vertices of the complement of $I$ that have a n.n. in $I$.

A contour $\Gamma_{i}$ of the configuration $\sigma$ is the subgraph defined by the set of vertices $V\left(\Gamma_{i}\right)=I_{i} \cup \partial_{E} I_{i}$ and all bonds of $\mathcal{T}_{k}$ between them. The interior $\operatorname{Int}\left(\Gamma_{i}\right)$ of $\Gamma_{i}$ is the set $I_{i}$.

Each connected subgraph $\Gamma_{i}=\left\{V\left(\Gamma_{i}\right), E\left(\Gamma_{i}\right)\right\}(i=1, \ldots, n)$, where $V\left(\Gamma_{i}\right)=I_{i} \cup \partial_{E} I_{i}$ and $E\left(\Gamma_{i}\right)$ are all the edges between n.n. pairs of points in $V\left(\Gamma_{i}\right)$, is called a contour of the configuration $\sigma$.

Obviously, there is a one-to-one correspondence between the configurations and the sets of all its contours, provided the boundary condition $\sigma^{D}$ is given. Every collection of contours thus constructed is called a compatible collection.

This allows to write the partition function in a finite ball $V_{r}$ of radius $r$ as follows:

$$
Z\left(V_{r}\right)=\sum_{\sigma} \exp \left\{-\beta\left[H(\sigma)-H\left(\sigma^{D}\right)\right]\right\}=\sum_{\left\{\Gamma_{1}, \ldots, \Gamma_{n}\right\}_{c}} \prod_{i=1}^{n} \varrho\left(\Gamma_{i}\right)
$$

with $\varrho(\Gamma)=\exp \left\{-\beta\left[H\left(\sigma_{\Gamma}\right)-H\left(\sigma^{D}\right)\right]\right\}$. Here the first sum is over all configurations that coincide with the configuration $\sigma^{D}$ outside the ball $V_{r}$, the second sum runs over all compatible families of contours with vertex set in $V_{r}$, and $\sigma_{\Gamma}$ denotes the configuration that contains only one contour $\Gamma$.

The two following lemmas provide the energy and entropy estimates for contours.

Lemma 4 For any contour $\Gamma$ the excess energy satisfies

$$
H\left(\sigma_{\Gamma}\right)-H\left(\sigma^{D}\right) \geq 2 J\left[(k+1)-2\left(d_{D}+1\right)\right]|\operatorname{Int}(\Gamma)| .
$$


Proof. We prove (2) by induction. If Int $(\Gamma)$ consists of one point, then $H\left(\sigma_{\Gamma}\right)-H\left(\sigma^{D}\right) \geq 2 J\left[(k+1)-2 d_{D}\right]$. Let $\Gamma=\{V(\Gamma), E(\Gamma)\}$, where $V(\Gamma)=$ $I \cup \partial_{E} I$, and suppose that the root $0 \in I$. Let $x \in I, x \neq 0$ be such a site that all the $k$ offsprings of $x$ do not belong to $I$. Note that the configuration $\sigma_{\Gamma}^{x}$, obtained from $\sigma_{\Gamma}$ by flipping a spin at $x$, also has one contour, and so $\sigma_{\Gamma}^{x}=$ $\sigma_{\Gamma^{\prime}}$, and Int $\left(\Gamma^{\prime}\right)=I \backslash x$. The contribution of the site $x$ to the energy $-H\left(\sigma_{\Gamma}^{x}\right)$ is at least $J\left[(k+1)-2\left(d_{D}+1\right)\right]$; indeed, the site $x$ in the configuration $\sigma_{D}$ has at most $d_{D}$ frustrated bonds, while the number of bonds connecting $x$ to $I \backslash x$ (which also can be frustrated) is one. Since the contribution of the site $x$ to the energy $H\left(\sigma_{\Gamma}\right)$ is exactly the negative of its contribution to $-H\left(\sigma_{\Gamma}^{x}\right)$, we have

$$
H\left(\sigma_{\Gamma}\right)-H\left(\sigma_{\Gamma}^{x}\right) \geq 2 J\left[(k+1)-2\left(d_{D}+1\right)\right]
$$

The following lemma is well-known (see e.g. [O]).

Lemma 5 Let $G$ be a graph of maximal degree $k+1$. Then the number of connected subgraphs $\Gamma \subset G$ with $n$ bonds, containing a given vertex is bounded from above by

$$
(k+1)^{2 n}
$$

With these two estimates, we have good control of cluster expansions for partition functions and correlation functions at low temperatures. These expansions allow to prove by known arguments that the states $\mu^{D_{+}}$and $\mu^{D_{-}}$ are extremal.

Acknowledgement 6 We are grateful to A. van Enter, P. Bleher and $U$. Rozikov for helpful discussions and comments.

\section{References}

[BRZ] Bleher, P. M.; Ruiz, J.; Zagrebnov, V. A. On the purity of the limiting Gibbs state for the Ising model on the Bethe lattice. J. Statist. Phys. 79 (1995), no. 1-2, 473-482.

[BG] Blekher, P. M.; Ganikhodzhaev, N. N. Pure phases of the Ising model on Bethe lattices. (Russian) Teor. Veroyatnost. i Primenen. 35 (1990), no. 2, 220-230; translation in Theory Probab. Appl. 35 (1990), no. 2, 216-227 (1991) 
[DS] R.L. Dobrushin and S. Shlosman: The problem of translationinvariance of Gibbs states at low temperatures, Mathematical physics reviews, Vol. 5, 53-195, Soviet Sci. Rev. Sect. C: Math. Phys. Rev., 5, Harwood Academic Publ., Chur, 1985.

[O] O. Ore, The-Four Color Problem. Academic Press, New-York, London, 1967.

[RR] Rozikov, U.A.; Rakhmatullaev, M.M. Description of weakly periodic Gibbs measures for the Ising model on a Cayley tree. (English), Theor. Math. Phys. 156, No. 2, 1218-1227 (2008); translation from Teor. Mat. Fiz. 156, No. 2, 292-302 (2008). 